\title{
Full idempotents in Leavitt path algebras
}

\author{
Ekrem Emre \\ Department of Mathematics, Duzce University \\ Konuralp Campus Duzce 81620, Turkey \\ ekrememre@duzce.edu.tr \\ Received 10 August 2017 \\ Accepted 13 March 2018 \\ Published 7 June 2018 \\ Communicated by P. Ara
}

\begin{abstract}
We give necessary and sufficient conditions on a directed graph $E$ for which the associated Leavit path algebra $L_{K}(E)$ has at least one full idempotent. Also, we define $E_{n}, n \geq 0$ sub-graphs of $E$ and show that $L_{K}(E)$ has at least one full idempotent if and only if there is a sub-graph $E_{r}$ such that the associated Leavitt path algebra $L_{K}\left(E_{r}\right)$ has at least one full idempotent.
\end{abstract}

Keywords: Full idempotent; Leavitt path algebra; restriction graph; Morita invariant property; source elimination.

Mathematics Subject Classification: 16S99, 16D90, 05C25

\section{Introduction}

In [10, Theorem 3], it was shown that, for any directed graph $E$ and for any commutative ring $R$ with identity, there is a subset $V \subset E^{0}$ by which we can define a Leavitt path algebra such that it is Morita equivalent to $L_{R}(E)$. In this paper, for any directed graph $E$ and any field $K$, we give necessary and sufficient conditions for which there is subset $V \subset E^{0}$ by which we can define a full idempotent in $L_{K}(E)$.

Before explaining the idea behind this work, we give some definitions. Let $R$ be any ring. For any idempotent $e \in R$, the ring $e R e$ is said to be a corner ring of $R$. Clearly $e R e$ is a ring with identity $e$. If $R e R=R$, then $e$ is said to be a full idempotent in $R$. Let $E$ be a directed graph and $K$ any field, then Leavitt path algebra associated to $E$ and $K$ is denoted by $L_{K}(E)$. In the Preliminaries section, we give necessary information on Leavitt path algebras (for more information and relevant terminology on Leavitt path algebras, see [3, 4]). Now here is the idea behind this work: Suppose that $P$ and $Q$ are two properties defined on rings such that, for any ring $R$ with identity, if $R$ satisfies $P$, then it does $Q$. Now assume 
that $P$ and $Q$ are Morita invariant properties (for more information on Morita invariant properties, see 13, 14). In this case even if $R$ does not have an identity but has a full idempotent $e$ and satisfies $P$, then it satisfies $Q$. To see that recall, by [1] Corollary 4.3], that $R$ and $e R e$ are Morita equivalent rings. Since $P$ is Morita invariant and $e R e$ is a ring with identity $e, e R e$ satisfies $P$ and so does $Q$. Therefore since $Q$ is Morita invariant, $R$ satisfies $Q$. Hence, if the properties $P$ and $Q$ are Morita invariant, then instead of having a unit to satisfy $Q$, it is sufficient for $R$ to satisfy $Q$ that $R$ has a full idempotent. So we can use that for the generalization such as in [7] Corollary 1.2].

This paper is divided into three sections. In the Preliminaries section, we give some graph-theoretic definitions and properties. Then in the main section of this paper, we define equivalence vertices, maximal vertices, maximal set of a hereditary subset $H \subseteq E^{0}$ and the set $\mathcal{H}^{E}$ which consists of hereditary subsets having some properties and, as the first main result of this paper, we prove that, for any directed graph $E$ and any field $K, L_{K}(E)$ Leavitt path algebra has at least one full idempotent if and only if there is at least one $H \in \mathcal{H}^{E}$ such that the maximal set of $H$ is finite (Theorem 3.1). Then by using the restriction graphs defined in [8, p. 3], we define $E_{n}, n \geq 0$ subgraphs of $E$ (Definition 3.5 and prove the second main result of this paper that $L_{K}(E)$ has at least one full idempotent if and only if there is a sub-algebra $L_{K}\left(E_{r}\right), r \geq 0$ of $L_{K}(E)$ such that $L_{K}\left(E_{r}\right)$ has at least one full idempotent (Theorem [3.2). Thus, we simplify the problem whether or not $L_{K}(E)$ has a full idempotent. Furthermore, in Lemma 3.4, by using a different approach we prove that, for any directed graph $E$, if we denote by $F$ the directed graph obtained by applying source elimination to $E$, then Leavitt path algebra over $E$ is Morita equivalent to the Leavitt path algebra associated to the one over $F$. Finally, in the last section of this paper, we give some examples.

\section{Preliminaries}

We briefly recall some graph-theoretic definitions and properties. For more information see [2]. A (directed) graph $E=\left(E^{0}, E^{1}, r, s\right)$ consists of two arbitrary sets $E^{0}, E^{1}$ and maps $r, s: E^{1} \rightarrow E^{0}$. The elements of $E^{0}$ are called vertices and the elements of $E^{1}$ edges. If $s^{-1}(v)$ is a finite set for every $v \in E^{0}$, then the graph is called row-finite. A vertex for which $s^{-1}(v)$ is empty is called a sink, a vertex for which $r^{-1}(v)$ is empty is called a source and, a vertex $v \in E^{0}$ for which $\left|s^{-1}(v)\right|=\infty$ is called an infinite emitter. If $v$ is either a sink or an infinite emitter, we call it a singular vertex. If $v$ is not a singular vertex, we call it a regular vertex. A path $\mu$ in a graph $E$ is a sequence of edges $\mu=e_{1}, \ldots, e_{n}$ such that $r\left(e_{i}\right)=s\left(e_{i+1}\right)$ for $i=1, \ldots, n-1$. In this case, $s(\mu):=s\left(e_{1}\right)$ is the source of $\mu, r(\mu):=r\left(e_{n}\right)$ is the range of $\mu$, and $n$ is the length of $\mu$. If $\mu=e_{1} \ldots e_{n}$ is a path, then we denote by $\mu^{0}$ the set of its vertices, that is, $\mu^{0}=\left\{s\left(e_{1}\right), r\left(e_{i}\right)\right.$ for $\left.1 \leq i \leq n\right\}$. If $\mu$ is a path in $E$, and if $v=s(\mu)=r(\mu)$, then $\mu$ is called a closed path based at $v$. If $s(\mu)=r(\mu)$ and $s\left(e_{i}\right) \neq s\left(e_{j}\right)$ for every $i \neq j$, then $\mu$ is called a cycle. For $n \geq 2$, we define 
$E^{n}$ to be the set of paths of length $n$, and $E^{*}=\bigcup_{n>0} E^{n}$ the set of all paths. The Leavitt path algebra of a graph $E$ is defined as the following.

Let $E$ be any directed graph, and $K$ any field. The Leavitt path $K$-algebra $L_{K}(E)$ of $E$ with coefficients in $K$ is the $K$-algebra generated by a set $\left\{v \mid v \in E^{0}\right\}$ of pairwise orthogonal idempotents, together with a set of variables $\left\{e, e^{*} \mid e \in E^{1}\right\}$, which satisfy the following relations:

(1) $s(e) e=e r(e)=e$ for all $e \in E^{1}$.

(2) $r(e) e^{*}=e^{*} s(e)=e^{*}$ for all $e \in E^{1}$.

(3) $e^{*} e^{\prime}=\delta_{e, e^{\prime}} r(e)$ for all $e, e^{\prime} \in E^{1}$.

(4) $v=\sum_{\left\{e \in E^{1} \mid s(e)=v\right\}} e e^{*}$ for every regular vertex $v \in E^{0}$.

The elements of $E^{1}$ are called real edges, while for $e \in E^{1}$ we call $e^{*}$ a ghost edge. The set $\left\{e^{*} \mid e \in E^{1}\right\}$ will be denoted by $\left(E^{1}\right)^{*}$. We let $r\left(e^{*}\right)$ denote $s(e)$, and we let $s\left(e^{*}\right)$ denote $r(e)$. If $\mu=e_{1} \ldots e_{n}$ is a path, then we denote by $\mu^{*}$ the element $e_{n}^{*} \ldots e_{1}^{*}$ of $L_{K}(E)$.

Specifically, we define a relation $\geq$ on $E^{0}$ by setting $v \geq w$ if there is a path $\mu \in E^{*}$ with $s(\mu)=v$ and $r(\mu)=w$. A subset $H$ of $E^{0}$ is called hereditary if $v \geq w$ and $v \in H$ imply $w \in H$. Denote by $\mathcal{H}_{E}$ the set of hereditary subsets of $E^{0}$. A hereditary set is saturated if every regular vertex which feeds into $H$ and only into $H$ is again in $H$, that is, if $v$ is a regular vertex such that $s^{-1}(v) \neq \emptyset$ and $r\left(s^{-1}(v)\right) \subseteq H$, then necessarily $v \in H$.

The hereditary saturated closure of a set $X$ of vertices is defined as the smallest hereditary and saturated subset of $E^{0}$ containing $X$. In [9, Remark 3.1], it was shown that the hereditary saturated closure of a set $X$ of vertices is $\bar{X}=\bigcup_{n=0}^{\infty} \Lambda_{n}(X)$, where $\Lambda_{0}(X)=T(X)=\left\{v \in E^{0}: x \geq v\right.$ for some $\left.x \in X\right\}$, and $\Lambda_{n}(X)=\left\{y \in E^{0}: 0<\left|s^{-1}(y)\right|<\infty\right.$ and $\left.r\left(s^{-1}(y)\right) \subseteq \Lambda_{n-1}(X)\right\} \cup \Lambda_{n-1}(X)$, for $n \geq 1$, where the set $T(X)$ is called tree of $X$ and clearly the smallest hereditary subset of $E^{0}$ containing $X$.

If $E^{0}$ is finite, then we have $\sum_{v \in E^{0}} v=1$; otherwise, $L_{K}(E)$ is a ring with a set of local units consisting of sums of distinct vertices. Conversely, if $L_{K}(E)$ is unital, then $E^{0}$ is finite.

\section{Full Idempotents in Leavitt Path Algebras}

Before proving the first main result of this paper, we need some definitions and remarks.

Definition 3.1. Let $E$ be a directed graph. If for two vertices $u$ and $v$, it is true that $u \geq v$ and $v \geq u$, then we call $u$ and $v$ as equivalence vertices and denote by $u \approx v$.

It is straightforward to show that " $\approx$ " is an equivalence relation on $E^{0}$.

Definition 3.2. Let $E$ be a directed graph and let $X \subseteq E^{0}$. If there is a vertex $u$ in $X$ such that whenever $v \geq u$, so $u \geq v$ for all $v \in X$, then $u$ is called maximal in $X$. 
Moreover, if $H \subseteq E^{0}$ is hereditary subset, then the maximal set of $H$ is defined as:

$$
\vec{H}=\{[u]: u \text { is maximal in } H\} .
$$

\section{Example 3.1.}

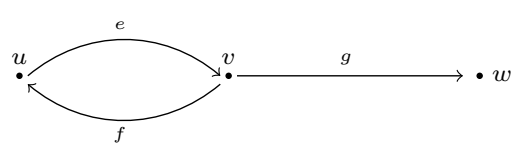

Fig. 1. E.

For the graph above, we have $u \approx v$ and $\overrightarrow{E^{0}}=\{[u]\}$.

Definition 3.3. Let $E$ be a directed graph. We define the set $\mathcal{H}^{E}$ as:

$$
\mathcal{H}^{E}=\left\{H \in \mathcal{H}_{E}: H=\bigcup_{[u] \in \vec{H}} T(u) \text { and } \bar{H}=E^{0}\right\} \text {. }
$$

It is clear that if $u \approx v$, then $T(u)=T(v)$. So the definition above is well defined.

Remark 3.1. Let $E$ and $F$ be directed graphs and $K$ any field. Then for any subset $X$ of $E^{0}$, we will denote by $I(X)$ the ideal of $L_{K}(E)$ generated by $X$. If $L_{K}(E)$ is Morita equivalent to $L_{K}(F)$, then we will briefly denote that by $L_{K}(E) \approx_{M} L_{K}(F)$.

Remark 3.2. Let $E$ be a directed graph and suppose that $H \in E^{0}$ is a hereditary subset. Then since $T(H)$ is the smallest hereditary set containing $H$ and $H$ is hereditary subset of $E^{0}$, we get that $T(H) \subseteq H, H \subseteq T(H)$ and so $T(H)=H$.

Remark 3.3. Let $E$ be e directed graph. If $H$ is a subset of $E^{0}$, then $\bar{H}=I(H) \cap$ $E^{0}$. (See the proof of [8, Lemma 2.1])

Now we are ready to prove the first main result of this article.

Theorem 3.1. Let $E$ be a directed graph and $K$ a field. Then $L_{K}(E)$ has at least one full idempotent if and only if there is a set $H \in \mathcal{H}^{E}$ whose maximal set is finite.

Proof. If $L_{K}(E)$ has a full idempotent $e$, then by [3, Lemma 1.6], there is an idempotent $u \in L_{K}(E)$ such that $u=\sum_{w \in W} w$ and $u e=e u=e$, where $W$ is a finite subset of $E^{0}$. If we define the set $H=T(W)=\bigcup_{w \in W} T(w)$, then since $W$ is finite, for every vertex $w \in W$, there is a maximal vertex $v$ in $W$ such that $w \in T(v)$. Therefore $H=\bigcup_{[v] \in \vec{H}} T(v)$ and clearly $\vec{H}$ is finite. Now we show that $\bar{H}=E^{0}$. Since $u \in I(H)$ and $e$ is a full idempotent in $L_{K}(E)$, we have $e \in I(H)$ and $L_{K}(E)=I(e) \subseteq I(H)$. Therefore $I(H)=L_{K}(E)$ and so $\bar{H}=I(H) \cap E^{0}=E^{0}$.

Conversely, suppose there is a set $H \in \mathcal{H}^{E}$ with a finite maximal set $V=$ $\left\{v_{1}, v_{2}, \ldots, v_{n}\right\}$. If $I(V)$ is the ideal generated by $V$, then by the assumption on 
$H, I(V)=I(H)=L_{K}(E)$. If $u=v_{1}+v_{2}+\cdots+v_{n}$, then $u$ is an idempotent and $u v_{i}=v_{i}=v_{i} u$ and so $I(u)=I(V)=L_{K}(E)$. Thus, $u$ is a full idempotent in $L_{K}(E)$.

Corollary 3.1. If e is a full idempotent in $L_{K}(E)$, then there is a full idempotent $u$ which is a sum of vertices such that $I(u)=I(e)$.

As a result of [12] Lemma 12], we can give the following lemma.

Lemma 3.1. Let $E$ be a directed graph and $H \subseteq E^{0}$ hereditary subset. If $u \in \bar{H}$ and $u$ is a base for a closed path, then $u \in H$.

Also by Theorem 3.1 and by Lemma [3.1] we can give the following two corollaries.

Corollary 3.2. Let $E$ be a directed graph and $K$ a field. If $E^{0} \notin \mathcal{H}^{E}$ and, for every $H$ hereditary proper subset of $E^{0}$, there is a vertex $u$ which is base of a closed path such that $u \notin H$, then $L_{K}(E)$ has no full idempotent.

Proof. Assume that $E^{0} \notin \mathcal{H}^{E}$ and, for every $H \subset E^{0}$ hereditary proper subset there is at least one vertex $u$ which is base of a closed path such that $u \notin H$. Now suppose that $\mathcal{H}^{E} \neq \emptyset$. Then there is at least one $H \subseteq E^{0}$ hereditary subset such that $\bar{H}=E^{0}$. Then by the assumption, we have $H \neq E^{0}$. Therefore $H$ must be a proper subset of $E^{0}$. But since for every proper hereditary subset $H$ of $E^{0}$, there is a vertex $u$ which is the base of a closed path such that $u \notin H$, by Lemma 3.1 . we get that $\bar{H} \neq E^{0}$ and so $H \notin \mathcal{H}^{E}$, a contradiction. Therefore, we must have $\mathcal{H}^{E}=\emptyset$ and then by Theorem $\left[3.1, L_{K}(E)\right.$ has no full idempotent.

Corollary 3.3. Let $E$ be a directed graph and $K$ a field. If there are infinitely many nonequivalent maximal vertices which are base of a closed path, then $L_{K}(E)$ has no full idempotent.

Proof. Suppose that there are infinite number of nonequivalent maximal vertices in $E$ which are the base of a closed path and denote by $M$ the set of those vertices. If we assume that $L_{K}(E)$ has at least one full idempotent, then we can find a finite set $V$ with similar way in the proof of Theorem 3.1. Since $\bar{V}=E^{0}$ and by Lemma 3.1 . for every $u \in M$ it is true that $u \in T(V)$ and so there must be at least one $v \in V$ such that $v \geq u$. Hence because $u$ is maximal, we get that $u \geq v$ and so $u \approx v$. Therefore if $u \in M$, then there is a vertex $v \in V$ such that $u$ is equivalent to $v$. But this is impossible because $V$ is a finite set and $M$ consists of infinite number of nonequivalent vertices. Therefore $L_{K}(E)$ must not have any full idempotent.

Now we recall restriction graph defined in [8] p. 3]. Let $E$ be a directed graph and let $H$ be hereditary subset of $E^{0}$. Then the restriction graph of $E$ over $H$ is defined as:

$$
E_{H}=\left(H,\left\{e \in E^{1} \mid s(e) \in H\right\}, r_{\left(E_{H}\right)^{1}}, s_{\left(E_{H}\right)^{1}}\right)
$$


Proposition 3.1. Let $E$ be a directed graph and $H \subseteq J \subseteq K$ hereditary subsets of $E^{0}$. If hereditary saturated closure of $H$ according to $E_{J}$ is $J$ and that of $J$ according to $E_{K}$ is $K$, then that of $H$ according to $E_{K}$ is $K$.

Proof. We shall be using [6, Lemma 1.4] and the idea in its proof, namely, if $X \subseteq E^{0}$ is a nonempty hereditary set, then $Y \subseteq E^{0}$ is contained in the saturated closure $\bar{X}$ of $X$ if and only if, for any regular vertex $u \in Y$, there is an integer $n \geq 0$ such that every path $p$ in $E$ with $s(p)=u$ and length $\geq n$ satisfies $r(p) \in X$. In particular, $r(q) \in X$ if $q$ is any path of length exactly $n$ in $E$ with $s(q)=u$. We will also be using the fact that if $u$ is any singular vertex in $Y$, then already $u \in X$. Consequently, all the singular vertices in $K=\bar{J}$ belong to $J$ and are singular in $J$ (as $J$ is hereditary) and since $J=\bar{H}$, they all belong to $H$. In order to show that $\bar{H}=K$, let $u$ be a regular vertex in $K$. Since $\bar{J}=K$ in $E_{K}$, there is an integer $m \geq 0$ such that every path $p$ in $E_{K}$ with $s(p)=u$ and length $m$ satisfies that $r(p) \in J$. If $r(p)$ is singular, then already $r(p) \in H$. So consider only the paths $p$ of length $m$, where $s(p)=u$ and $r(p)$ is regular and belongs to $J \backslash H$. In particular, since $H$ is hereditary, every vertex in all these paths of length $m$ must be regular. A simple induction on $m$ then shows that there are only finitely many paths $p$ of length $m$ with $s(p)=u$ and $r(p) \in J \backslash H$. Let $p_{1}, \ldots, p_{k}$ be a listing of these paths of length $m$ with $r\left(p_{i}\right)=v_{i} \in J, i=1, \ldots, k$. Since $J=\bar{H}$, for each $v_{i}$, there is an integer $n_{i} \geq 0$ such that all paths $q$ with $s(q)=v_{i}$ and length $\geq n_{i}$ satisfy $r(q) \in H$. Let $n=\max \left\{n_{i}: i=1, \ldots, k\right\}$. Then every path $\mu$ in $E_{K}$ with $s(\mu)=u$ and length $\geq m+n$ satisfies $r(\mu) \in H$. By Lemma 1.4 of [6], $K=\bar{H}$.

Also by [6] Lemma 1.4], we can give the following lemma.

Lemma 3.2. Let $E$ be a directed graph and let $H$ be a proper hereditary subset of $E^{0}$. Then $\bar{H}=E^{0}$ if and only if, for every $u \in E^{0}$, there is a positive integer $n_{u}$ such that all paths which emit from $u$ and whose length are at least $n_{u}$ connect to $H$.

Now we modify the source elimination process defined in [5, Definition 1.2].

Definition 3.4. Let $E=\left(E^{0}, E^{1}, r, s\right)$ be a directed graph. Denote by $S$ the set of all regular sources in $E$ and, define the following set:

$$
s^{-1}(S)=\left\{e \in E^{1}: e \in s^{-1}(v), v \in S\right\} .
$$

Then we form the source elimination graph $E_{\backslash S}$ of $E$ as follows:

$$
\begin{aligned}
& E_{\backslash S}^{0}=E^{0} \backslash S, \\
& E_{\backslash S}^{1}=E^{1} \backslash s^{-1}(S), \\
& s_{E_{\backslash S}}=\left.s\right|_{E_{\backslash S}^{1}}, \\
& r_{E_{\backslash S}}=\left.r\right|_{E_{\backslash S}^{1}} .
\end{aligned}
$$

Here we define $E_{n}, n \geq 0$ subgraphs, which we use to describe second main result of this paper, of $E$. 
Definition 3.5. Let $E$ be a directed graph. For $n \geq 0$, we define the sub-graphs $E_{n}$ of $E$ as: $E_{0}=E$ and if $\mathcal{H}^{E_{n-1}} \neq \emptyset, n \geq 1$, then take any $H_{n-1} \in \mathcal{H}^{E_{n-1}}$, denote by $G_{n-1}$ the restriction graph of $E_{n-1}$ over $H_{n-1}$ and take $E_{n}$ as the result graph of applying source elimination to $G_{n-1}$.

In [5. Proposition 3.1, it was shown that removing sources from a row-finite graph would give a Leavitt path algebra Morita equivalent to the one associated to the original graph, and in [11], this result was generalized to any directed graph $E$. Now in Lemma 3.3, we prove that by using a different approach.

Lemma 3.3. Let $E$ be a directed graph. If $F$ is the result graph of applying source elimination to $E$, then $\overline{F^{0}}=E^{0}$ and $L_{K}(E) \approx_{M} L_{K}(F)$.

Proof. If we denote by $S \subseteq E^{0}$ the set of regular sources of $E$, then by Definition [3.4, we have $S \cup F^{0}=E^{0}$ and $S=\left\{y \in E^{0}: 0<\left|s^{-1}(y)\right|<\infty, r\left(s^{-1}(y)\right) \subseteq\right.$ $\left.F^{0}, y \notin F^{0}\right\}$. Therefore since $F^{0}$ is a hereditary subset of $E^{0}, \Lambda_{1}\left(F^{0}\right)=\left\{y \in E^{0}\right.$ : $\left.0<\left|s^{-1}(y)\right|<\infty, r\left(s^{-1}(y)\right) \subseteq \Lambda_{0}\left(F^{0}\right)\right\} \cup \Lambda_{0}\left(F^{0}\right)=\left\{y \in E^{0}: 0<\left|s^{-1}(y)\right|<\right.$ $\left.\infty, r\left(s^{-1}(y)\right) \subseteq F^{0}\right\} \cup F^{0} \supseteq S \cup F^{0}=E^{0}$ and then $\overline{F^{0}}=E^{0}$. Since $E^{0}=\overline{F^{0}}=$ $I\left(F^{0}\right) \cap E^{0}$, we have $E^{0} \subseteq I\left(F^{0}\right)$ and then $I\left(F^{0}\right)=L_{K}(E)$. Hence by [8, Lemma 2.4], we get that $L_{K}(F)=L_{K}\left(E_{F^{0}}\right) \approx_{M} I\left(F^{0}\right)=L_{K}(E)$.

Lemma 3.4. If we define a property $P$ over any ring $R$ as: " $R$ has at least one full idempotent", then $P$ is Morita invariant.

Proof. Let $R$ be a ring such that it satisfies $P$. If $e \in R$ is a full idempotent, then since the corner ring $e R e$ has an identity, $e$ Re has at least one full idempotent. Therefore by [13. Corollary 18.35], $P$ is Morita invariant.

Now we are ready to prove the second main result of this paper, so we restrict the problem of finding full idempotent in $L_{K}(E)$ to subalgebra $L_{K}\left(E_{r}\right), r \geq 0$ of $L_{K}(E)$.

Theorem 3.2. Let $E$ be a directed graph and $K$ a field. Then $L_{K}(E)$ has at least one full idempotent if and only if there is a directed subgraph $E_{r}, r \geq 0$ of $E$ such that $L_{K}\left(E_{r}\right)$ has at least one full idempotent.

Proof. If $L_{K}(E)$ has at least one full idempotent, then $r=0$. Now suppose that there is a subgraph $E_{r}, r \geq 0$ such that $L_{K}\left(E_{r}\right)$ has a full idempotent. If $r=0$, then by $E_{0}=E$, we have $L_{K}(E) \approx_{M} L_{K}\left(E_{0}\right)$. Suppose that $r \geq 1$. Then for $\left(E_{r}\right)^{0} \subseteq\left(G_{r-1}\right)^{0} \subseteq\left(E_{r-1}\right)^{0}$, we have $G_{r-1}=E_{H_{r-1}}$ and then $\left(G_{r-1}\right)^{0}=H_{r-1}$. So we get that $\overline{\left(G_{r-1}\right)^{0}}=\overline{\left(H_{r-1}\right)}=\left(E_{r-1}\right)^{0}$ and, by Lemma 3.4 the saturated closure of $\left(E_{r}\right)^{0}$ according to $G_{r-1}$ is $\left(G_{r-1}\right)^{0}$. Therefore by Proposition 3.1] the saturated closure of $\left(E_{r}\right)^{0}$ according to $E_{r-1}$ is $\left(E_{r-1}\right)^{0}$. By continuing to repeat this process, we get that the saturated closure of $\left(E_{r}\right)^{0}$ according to $E_{0}=E$ is $E^{0}$. Therefore since $E^{0}=\overline{\left(E_{r}\right)^{0}}=I\left(\left(E_{r}\right)^{0}\right) \cap E^{0}$, we get that $E^{0} \subseteq I\left(\left(E_{r}\right)^{0}\right)$ and then $I\left(\left(E_{r}\right)^{0}\right)=L_{K}(E)$. Hence by [8, Lemma 2.4.], $L_{K}\left(E_{r}\right)=L_{K}\left(E_{\left(E_{r}\right)^{0}}\right) \approx_{M}$ 
$I\left(\left(E_{r}\right)^{0}\right)=L_{K}(E)$ and so by Lemma 3.4, we get that $L_{K}(E)$ has a full idempotent, as desired.

\section{Examples}

\section{Example 4.1.}

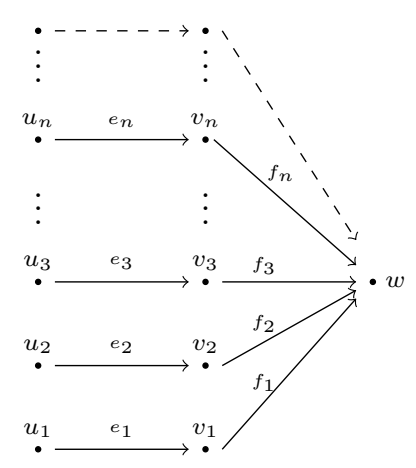

Fig. 2. $E$.

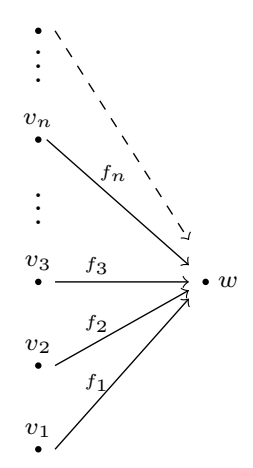

Fig. 3. $E_{1}$.
- $w$

Fig. 4. $E_{2}$.

Take $\overrightarrow{E^{0}}:=\left\{\left[u_{1}\right],\left[u_{2}\right], \ldots,\left[u_{n}\right], \ldots\right\}$ and then $E^{0} \in \mathcal{H}^{E}$. Therefore $H=E^{0}$ and $G_{0}=E_{E^{0}}=E$. Then by applying source elimination to $E$, we get $E_{1}$. Now take $\overrightarrow{\left(E_{1}\right)^{0}}:=\left\{\left[v_{1}\right],\left[v_{2}\right], \ldots,\left[v_{n}\right], \ldots\right\}$ and then $\left(E_{1}\right)^{0} \in \mathcal{H}^{E_{1}}$. Similarly by applying source elimination to $E_{1}$, we get $E_{2}$. Since $\left(E_{2}\right)^{0}$ is finite, $L_{K}\left(E_{2}\right)$ has an identity. Therefore $L_{K}\left(E_{2}\right)$ has at least one full idempotent and then by Theorem $3.2 L_{K}(E)$ has at least one full idempotent.

\section{Example 4.2.}

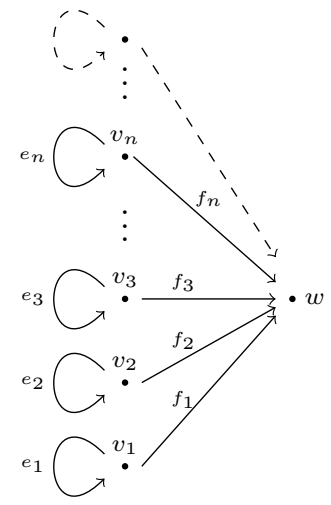

Fig. 5. $E$.

Since $E$ has infinite number of nonequivalent maximal vertices which are base of loops, by Corollary $3.3 L_{K}(E)$ has no full idempotent. 


\section{Example 4.3.}

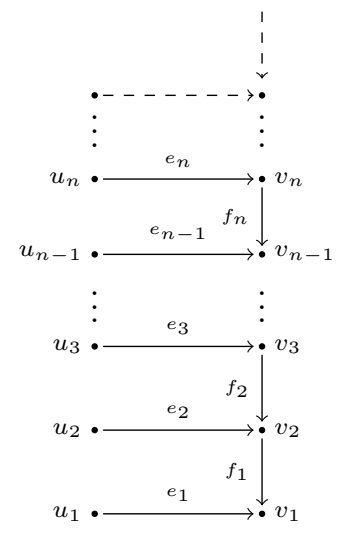

Fig. 6. E.

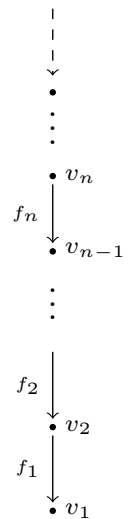

Fig. 7. $E_{1}$.

Take $\overrightarrow{E^{0}}:=\left\{\left[u_{1}\right],\left[u_{2}\right], \ldots,\left[u_{n}\right], \ldots\right\}$ and then $E^{0} \in \mathcal{H}^{E}$. Therefore $H=E^{0}$ and $G_{0}=E_{E^{0}}=E$. Then by applying source elimination to $E$, we get $E_{1}$. Now take $H_{1}:=\left\{v_{1}\right\}$ and then by Lemma 3.2 , we have $H_{1} \in \mathcal{H}^{E_{1}}$ and $\overrightarrow{H_{1}}$ is finite. Then by Theorem 3.1, $L_{K}\left(E_{1}\right)$ has at least one full idempotent and then by Theorem 3.2 $L_{K}(E)$ has at least one full idempotent.

\section{References}

[1] G. Abrams, Morita equivalences for rings with local units, Comm. Algebra 11(8) (1983) 801-837.

[2] G. Abrams, P. Ara and M. Siles Molina, Leavitt Path Algebras, Lecture Notes in Mathematics, Vol. 2191 (Springer, 2017).

[3] G. Abrams and G. Aranda Pino, The Leavitt path algebra of a directed graph, J. Algebra 293(2) (2005) 319-334.

[4] G. Abrams and G. Aranda Pino, The Leavitt path algebra of arbitrary graphs, Houston J. Math. 34(2) (2008) 423-442.

[5] G. Abrams, A. Louly, E. Pardo and C. Smith, Flow invariants in the classification of Leavitt path algebras, J. Algebra 333(1) (2011) 202-231.

[6] G. Abrams, K. M. Rangaswamy and M. Siles Molina, The Socle series of a Leavitt path algebra, Israel J. Math. 184 (2011) 413-435.

[7] P. Ara, The exchange property for purely infinite simple rings, Proc. Amer. Math. Soc. 132(9) (2004) 2543-2547.

[8] G. Aranda Pino, E. Pardo and M. Siles Molina, Exchange Leavitt path algebras and stable rank, J. Algebra 305(2) (2006) 912-936.

[9] T. Bates, J. H. Hong, I. Raeburn and W. Szymanski, The ideal structure of the C*-algebras of infinite graphs, Illinois J. Math. 46(4) (2002) 1159-1176.

[10] L. O. Clark, A. An Huef and P. Luiten-Apirana, Subsets of vertices give Morita equivalences of Leavitt path algebras, Bull. Aust. Math. Soc., doi: 10.1017/ S0004972717000247 (2017) 1-11. 
[11] L. O. Clark and A. Sims, Equivalent groupoids have Morita equivalent Steinberg algebras, Pure Appl. Algebra 219(6) (2015) 2062-2075.

[12] P. Colak, Two sided ideals in Leavitt path algebras, J. Algebra Appl. 10 (2010) 801-809.

[13] T. Y. Lam, Lectures on Modules and Rings (Springer-Verlag New York, Berlin, Heidelberg, 1999).

[14] M. Siles Molina and J. F. S. Hernández, Morita equivalence and morita invariant properties: Applications in the context of Leavitt path algebras, in Non-Associative and Non-Commutative Algebra and Operator Theory, eds. C. T. Gueye and M. S. Molina, Vol. 160 (Springer, Cham, 2016), pp. 83-103. 